\title{
DARNING HERNIOPLASTY VS. MESH HERNIOPLASTY- A NON-RANDOMISED CONTROLLED TRIAL WITH 3 YEARS FOLLOWUP IN A TERTIARY CARE HOSPITAL, RAJAHMUNDRY, ANDHRA PRADESH, INDIA
}

\author{
Ganni Bhaskar Rao ${ }^{1}$, K. L. Narasimha Rao², Samir Ranjan Nayak3, Lavanya K. M4, P. Ashrith ${ }^{5}$
}

${ }^{1}$ Associate Professor, Department of General Surgery, GSL Medical College, Rajahmundry, Andhra Pradesh. ${ }^{2}$ Associate Professor, Department of General Surgery, GSL Medical College, Rajahmundry, Andhra Pradesh.

${ }^{3}$ Associate Professor, Department of General Surgery, GSL Medical College, Rajahmundry, Andhra Pradesh.

${ }^{4}$ Assistant Professor, Department of Community Medicine, GSL Medical College, Rajahmundry, Andhra Pradesh.

${ }_{5}^{5}$ Senior Resident, Department of General Surgery, GSL Medical College, Rajahmundry, Andhra Pradesh.

ABSTRACT

\section{BACKGROUND}

Inguinal hernias are protrusions of abdominal cavity contents through the inguinal canal. Inguinal hernias are the commonest of all hernias. Surgery is the definitive treatment for hernia. This study aims to compare the results of costly, yet popular Lichtenstein mesh plasty with darning technique for inguinal hernia.

\section{MATERIALS AND METHODS}

Study Design and Setting: A non-randomised controlled trial with 100 patients of inguinal hernia was conducted in a tertiary care hospital, Rajahmundry between May 2010 - May 2013 (3 years). All patients who attended the surgical OPD during May 2010 to May 2013 (3 years) and satisfied the inclusion criteria were included in the study. The patients were randomly allocated/ treated with darn hernioplasty and mesh hernioplasty alternatively. Both the techniques were compared in terms of duration of surgery, cost and postoperative complications. Results were expressed as percentages, mean and standard deviation. Independent $t$-test was used to analyse the difference among continuous variables. A P value of $<0.05$ is considered statistically significant.

\section{RESULTS}

Most of them $44(44 \%)$ were in the age group of 25 to 39 years. Mean age was 36 years with a standard deviation of $+/-2.41$. Hernia was seen more commonly among males, 98 (98\%). Indirect hernia was more common, 73 (73\%). Mean duration for darn repair was 40 minutes compared to 68 minutes for mesh repair. The difference in duration of surgery between two techniques was statistically significant ( $\mathrm{p}<0.05)$. The cost for mesh repair is more compared to darn repair. Following darn repair 6 (12\%) developed postoperative complications and most common being haematoma 3 (6\%), while with mesh repair 7 (14\%) had postoperative complications and most common being wound infection $3(6 \%)$.

\section{CONCLUSION}

Darn repair is superior to mesh repair of inguinal hernia in terms of cost effectiveness, duration of surgery with similar postoperative outcome.

\section{KEYWORDS}

Inguinal Hernia, Darn Repair, Mesh Repair, Comparison, Andhra Pradesh, India.

HOW TO CITE THIS ARTICLE: Rao GB, Rao KLN, Nayak SR, et al. Darning hernioplasty vs. mesh hernioplasty- a non-randomised controlled trial with 3 years followup in a tertiary care hospital, Rajahmundry, Andhra Pradesh, India. J. Evolution Med. Dent. Sci. 2017;6(86):5947-5950, DOI: 10.14260/jemds/2017/1294

\section{BACKGROUND}

Hernia term is derived from the Latin word for rupture. Inguinal hernia repair accounts for $10 \%-15 \%$ of all surgical procedures and constitutes $73 \%$ of all external hernia.(1,2) Surgery is indicated in many hernias to prevent complications. These patients must be thoroughly evaluated before hernia repair.(2) Fortunately, most inguinal hernias are repaired electively to prevent strangulation.(3)

'Financial or Other Competing Interest': None.

Submission 31-08-2017, Peer Review 12-10-2017,

Acceptance 19-10-2017, Published 26-10-2017.

Corresponding Author:

Dr. K. L. Narasimha Rao,

Department of General Surgery,

GSL Medical College and General Hospital,

Lakshmipuram, NH - 16, Rajahmundry-533296,

Andhra Pradesh.

E-mail: drklnarasimharao@hotmail.com

DOI: $10.14260 /$ jemds/2017/1294

\section{(c) $(7)$}

Gilbert in 1987 classified four basic techniques for hernia repair.(4) These include pure tissue repair, combined tissue and prosthetic repair, pure prosthetic repair and darn. It is doomed to failure unless rendered free tension. Many surgeons use darn as sole method for inguinal hernia repair.(5) A better result is obtained with Lichtenstein (mesh) repair, but is costly. Because of poor socioeconomic status, non-affordability of patients and non-availability of mesh in small cities darn technique was chosen to ascertain its effectiveness, postoperative complications and recurrence. The advantage of darn repair lies in the fact that it does not require mesh, so it is much less expensive.(6) This study is an attempt to compare two tension free techniques, darning versus mesh repair in cases of inguinal hernia with respect to operative time taken, postoperative complications, recurrence and cost benefit factors. 


\section{MATERIALS AND METHODS}

A non-randomised controlled trial with 100 patients of inguinal hernia was conducted in a tertiary care hospital, Rajahmundry. Inclusion Criteria: Patients with unilateral inguinal hernias, non-obstructive, reducible and primary were included in the study. Exclusion Criteria: All those who did not consent were excluded. Patients with bilateral hernia or presented with complications like obstruction, irreducible and recurrent hernia were also excluded from the study. Study by convenient sample technique, since the duration was short period. The sample sizes were selected by convenience sample technique.

\section{Sample Size}

A total of 127 patients with inguinal hernias attended the surgical OPD during 3 years, of which 111 patients could satisfy the inclusion criteria and only 100 patients could be taken as the whole sample for study, since 11 patients did not consent for the study. However, even those patients have been treated appropriately. The sample size estimation was also done at conveniences.

\section{Sampling}

All patients who attended the surgical OPD during May 2010 to May 2013 (3 years) and satisfied the inclusion criteria were included in the study. Every patient who satisfied the inclusion criteria and consented for the study was randomly allocated to two groups alternatively. Thus, the elimination of selection bias. First group was treated with darn hernioplasty and second with mesh hernioplasty. Both the techniques were compared in terms of duration of surgery, cost and postoperative complications. This study was carried out in Department of General Surgery, GSL General Hospital, Rajahmundry, Andhra Pradesh in between May 2010 - May 2013 (3 yrs.). The study protocol was approved by the Ethical Committee of the institution. A written consent was taken from the participants at the beginning of the study.

\section{Statistical Analysis}

The collected data was entered in Microsoft Excel, double checked for errors and analyses using Epi-Info software. Results were expressed as percentages, mean and standard deviation. Independent t-test was used to analyse the difference among continuous variables. A P value of $<0.05$ is considered statistically significant.

\section{Surgical Procedure}

All patients had to have their parameters like routine blood, blood sugar, serum urea, serum creatinine, chest x-ray, ECG and vitals checked. If all parameters were within normal limits, the patients could get clearance for surgery. Patient selection bias was eliminated strictly by the inclusion criteria being declared eligible for the study by three doctors. Before surgery, the repair procedures were explained to all patients and duly signed. Informed consent was obtained from all the patients. All patients received a single dose of Inj. Ceftriaxone $1 \mathrm{gm} \mathrm{IV} \mathrm{at} \mathrm{the} \mathrm{onset} \mathrm{of} \mathrm{anaesthesia} \mathrm{and} \mathrm{all} \mathrm{of} \mathrm{them} \mathrm{were}$ operated under spinal anaesthesia or epidural anaesthesia.

A skin crease approach following Langer's line was adopted in all cases. The incision was deepened, tackling subcutaneous neurovascular structures as usual till the external oblique aponeurosis was reached. The resulting slit was about an inch above the inguinal ligament, which provided a large lower leaf for optimal closure. A gentle sweeping action with the index finger under the aponeurosis helps to open this plane widely for an adequate darn or mesh insertion. The cremasteric fascia was always incised which helped mobilise the cord structures properly.

In the mesh group, a sheet of polypropylene mesh $(7.5 \mathrm{x}$ 15) was trimmed to fit the adequately dissected out space, with a slit cut laterally to accommodate the spermatic cord. The mesh overlapped the pubic tubercle by $1-2 \mathrm{~cm}$ medially and superiorly extended over the conjoint tendon to lie 2 - 3 $\mathrm{cm}$ lateral to internal ring. The mesh was then fixed in position by 2 - 0 prolene.

In the darn group, a 1-0 prolene suture was used to reconstruct the inguinal bed with tension-free darn. It was started with good strong bite off the tough tendinous structures near the pubic tubercle and emerging out through the lateral edge of the rectus sheath with bulky bite in between. The loosely interwoven bites continued laterally and the back-forming two to three rows of continuous stitches were placed in staggered manner to spread the tension between the fibres of the inguinal ligament. The Aberdeen knot was used to avoid a thick prolene knot at the end. A gap of $0.5-1 \mathrm{~cm}$ was maintained between the stitches to obtain a closely knit darn.

In both groups having checked for homeostasis meticulously and after replacing the safeguarded iliohypogastric nerve and cord structures, the external oblique aponeurosis was closed with 2-0 prolene interrupted sutures. The skin was opposed using 3-0 simple interrupted nylon sutures. Operative time taken from skin incision to skin closure was recorded in all cases of both groups.

Patients of both groups were routinely given oral analgesics, which they were advised to continue for $72 \mathrm{~h}$ into the postoperative period. All patients were observed for 72 hours and were discharged only if they feel comfortable and confident with themselves. The emergency communication protocol was explained to all patients and necessary advice was given to all on discharge. The first reviews on the patients of both groups were carried out on the 7th postoperative day. Detailed records were kept concerning their pain profile, return to normal activities and early postoperative complications like wound gape, scrotal indurations, testicular atrophy, neuralgia, mesh/darn infection requiring withdrawal and recurrence. Stitches were removed on the 7 th postoperative day. Patients were followed up at 1, 3, 6, 9, 12 and 18 months post operation and evaluated for any complications.

\section{RESULTS}

All patients were in the age group of 16 - 58 years. Most of them, $44(44 \%)$ were in the age group of 25 to 39 years. Mean age was 36 years with a standard deviation of $+/-2.41$. Hernia was seen more commonly among males, 98 (98\%). Indirect hernia was more common 73 (73\%) and direct hernia 27 (27\%). 


\section{TYPES OF HERNIA (PERCENTAGE)}

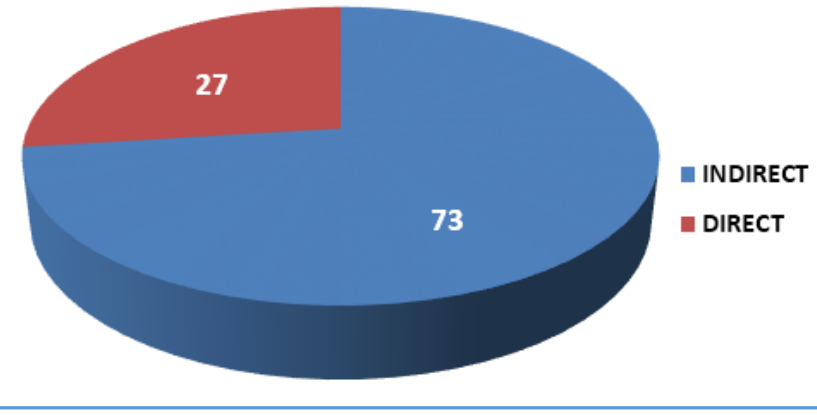

\begin{tabular}{|c|c|c|}
\hline Age Group & Number & Percentage \\
\hline$<25$ & 29 & $29 \%$ \\
\hline $25-39$ & 44 & $44 \%$ \\
\hline$>40$ & 27 & $27 \%$ \\
\hline \multicolumn{2}{|c|}{ Table 1. Age Distribution of the Study Population } \\
\hline
\end{tabular}

\section{Postoperative Complications}

Of the 50 patients who underwent darn repair 6 (12\%) developed postoperative complications, of which haematoma was most commonly seen in $3(6 \%)$ of patients followed by wound infection, recurrence and nerve paresis, each seen in 1 (2\%) of the patients. Among 50 patients who underwent mesh repair, 7 (14\%) had postoperative complications. Most common postoperative complication was wound infection 3 (6\%) followed by recurrence in 2 (4\%) patients and least common was haematoma $1(2 \%)$ and nerve paresis $1(2 \%)$. In 3 patients who had infection following mesh repair, mesh has been removed.

\begin{tabular}{|c|c|c|c|c|}
\hline \multirow{2}{*}{ Complications } & \multicolumn{2}{|c|}{ Darn (n= 50) } & \multicolumn{2}{c|}{ Mesh (n= 50) } \\
\cline { 2 - 5 } & Number & $\mathbf{\%}$ & Number & \% \\
\hline Wound infection & 1 & 2 & 3 & 6 \\
\hline Haematoma & 3 & 6 & 1 & 2 \\
\hline Recurrence & 1 & 2 & 2 & 4 \\
\hline Nerve paresis & 1 & 2 & 1 & 2 \\
\hline Total & $\mathbf{6}$ & $\mathbf{1 2}$ & $\mathbf{7}$ & $\mathbf{1 4}$ \\
\hline Table 2. Postoperative Complications in Two Groups \\
\hline
\end{tabular}

\section{Comparison of Cost for both Procedures}

At the current currency values and expenses, the cost for mesh repair is more as the expenditure for mesh is additional compared to darn repair. Mesh repair costs almost double the cost for darn repair, which is significantly considerable.

\begin{tabular}{|c|c|c|}
\hline Items & $\begin{array}{c}\text { Mesh Repair } \\
\text { (Rupees) }\end{array}$ & $\begin{array}{c}\text { Darn Repair } \\
\text { (Rupees) }\end{array}$ \\
\hline Investigations & 1200 & 1200 \\
\hline Suture material & 200 & 500 \\
\hline Mesh kit & 1500 & 0 \\
\hline $\begin{array}{c}\text { Antibiotics, anaesthesia } \\
\text { drugs }\end{array}$ & 1500 & 1000 \\
\hline Total & 4400 & 2700 \\
\hline \multicolumn{2}{|c|}{$\begin{array}{c}\text { Table 3. Comparison of Cost in Indian } \\
\text { Rupees (INR) for both Procedures }\end{array}$} \\
\hline
\end{tabular}

\section{Duration of Surgery}

Even in terms of duration of surgery for both procedures mesh repair consumes more time, about 60 - 80 minutes compared to darn repair of $35-50$ minutes. Mean duration for darn repair was $40+/-8$ minutes compared to $68+/-12$ minutes for mesh repair. The difference in duration of surgery between two techniques was statistically significant $(p<0.05$ ) with the mesh repair consuming significantly longer duration compared to darn repair.

\section{DISCUSSION}

The objective in inguinal hernia repair is to provide a tension free repair, when there is posterior inguinal wall weakness. As a primary outcome in our series, we recorded recurrence of 1 in darn and 2 in mesh repair till date. Complication rates in both groups were very minimal. However, what stands out in the study is the comparable outcome achieved with the darn procedure at such nominal operative costs when compared with mesh (Rs. 2000 - 2700/ patient for the darn repair; Rs. 4000 - 4500/ patient for mesh repair). Effective aspect of the darn (direct benefits) places it at a more favourable position than mesh in lower socioeconomic classes. A sizeable proportion of inguinal hernia patients from the lower socioeconomic group avoid operative treatment only because of non-affordability (cost), thereby increasing morbidity/ mortality rates. Thus, they end up having reduced quality adjusted life years (QALY) and being less productive to their employers and society at large (indirect benefits). Our only limitation is absence of a truly long followup period, i.e. 10 - 15 years. All our patients are still under surveillance and we hope to come up with detailed long-term followup results. Darn repair is easy to perform and can be used for primary (direct, indirect) hernias with weak posterior wall and recurrent hernia. In our study recurrence with darn repair was very low (2\%), which is comparable to the results of Moloney(7) with a recurrence as low as $0.8 \%$ with inguinal darn repair. Abrahamson $(8,5,6,9)$ was the first to point out the defect that could lead to high recurrence rate in darn repair. His series for more than 1000 repairs reported in 1995 recorded recurrence rate as low as $0.8 \%$. Lifschutz and Kingsnorth achieve similar result in recent times.(10,4) Koukourou A in 2001 and Chakraborty in 2007 compared the darn with mesh and reported no difference in early and late complications and achieved recurrence rate(2,3) comparable to our study. Similar to our study results showing darn repair with better results compared to mesh repair, Kingsnorth in 1992 and Thapar in 2000 had already achieved better result with darn technique in comparison with shouldice technique. $(4,1)$ In the present study, $6 \%$ patients in darn repair and $14 \%$ patients in mesh repair suffered from postoperative complication of haematoma, which is comparable to a study by Zedan $\mathrm{AM}(11)$ reporting the incidence of haematoma as $12.5 \%$ in darn repair and $8.3 \%$ in mesh repair. Similar findings were reported by Bhushan TV.(12)

\section{Limitation of the Study}

Due to short duration of study, convenience sample technique was followed. Thus, sample size was also calculated by convenience. The results of the study cannot be generalised due to the potential bias resulting from the sample technique and sample size estimation. 


\section{CONCLUSION}

Both the groups were compared for postoperative complications and it was found that there was no significant difference among the two groups. But cost-wise, the darn repair technique is more cost effective than mesh repair. Mesh repair is definitely the more popular version of the two, but in countries where cost factors still play a part and prevent many from seeking early treatment a wellconstructed darn is an equally effective and less costly treatment option with less duration of surgery and similar postoperative outcomes. Present study revealed that darn repair is superior to mesh repair of inguinal hernia in terms of cost effectiveness, duration of surgery with similar postoperative outcome. Hence, it could be considered for repair of inguinal hernias before thinking about mesh repair, especially in developing countries like India and among low socioeconomic groups.

\section{REFERENCES}

[1] Thapar V, Rao P, Deshpande A, et al. Shouldice's herniorrhaphy versus Moloney's darn herniorrhaphy in young patients: a prospective randomized study. J Postgrad Med 2000;46(1):9-12.

[2] Koukourou A, Lyon W, Rice J, et al. Prospective randomized trial of polypropylene mesh compared with nylon darn in inguinal hernia repair. Br J Surg 2001;88(7):931-4.
[3] Swarup C, Amitava M, Maitreyee B. Tension free inguinal hernia repair comparing darn with mesh: a prospective randomized controlled clinical trial. Indian J Surg 2007;69(2):52-6.

[4] Kingsnorth AN, Gray MR, Nott DM. Prospective randomized trial comparing shouldice technique and plication darn for inguinal hernia. $\mathrm{Br} \mathrm{J}$ Surg 1992;79(10):1068-70.

[5] Abrahamson J. Chapter on hernia. Maingot's abdominal operations. $10^{\text {th }}$ international edition. 2001:503-6.

[6] Abrahamson J. Hernias. In: Zinner MJ, Schwartz SI, Ellis H, (eds). Maingot's abdominal operations. $10^{\text {th }}$ edn. Connecticut: Appleton and Lange 1997:479-580.

[7] Moloney GE. Results of nylon darn repairs of herniae. Lancet 1958;1(7015):273-8.

[8] Abrahamson J. Factors and mechanisms leading to recurrence. Probl Gen Surg 1995;12:151-8.

[9] Abrahamson J. Etiology and pathophysiology of primary and recurrent groin hernia formation. Surg Clin North Am 1998;78(6):953-72.

[10] Lifshutz H, Juler GL. The inguinal darn. Arch Surg 1986;121(6):717-9.

[11] Zedan AM. Inguinal hernioplasty by darning vs. prosthetic mesh. Tikrit Med J 2009;15(1):158-62.

[12] Bhushan TV. Tension free inguinal hernia repair comparing darn with mesh: a prospective randomized controlled clinical trial. Indian J Surg 2007;69(5):215. 